\title{
Comparison of three non-invasive devices to assess the sagittal spinal configuration in an heterogeneous population of scoliotic and non scoliotic subjects Manuel Rigo* and M Villagrasa
}

\author{
Address: E. Salvá Spinal Deformities Rehabilitation Institute. Vía Augusta 185, 08021 Barcelona, Spain \\ Email: Manuel Rigo* - lolo_rigo@hotmail.com \\ * Corresponding author
}

\author{
from 4th International Conference on Conservative Management of Spinal Deformities \\ Boston, MA, USA. 13-16 May 2007 \\ Published: 12 October 2007 \\ Scoliosis 2007, 2(Suppl I):S35 doi:I0.II86/1748-7|6I-2-SI-S35
}

This abstract is available from: http://www.scoliosisjournal.com/content/2/SI/S35

(c) 2007 Rigo and Villagrasa; licensee BioMed Central Ltd.

\section{Objective}

To compare three non-invasive devices (SpineScan ${ }^{\circledR}$, Myrin $^{\circledast}$ inclinometer, Saunders ${ }^{\circledR}$ goniometer) to assess the sagittal postural curvatures of the spine (thoracic kyphosis, lumbar lordosis and pelvis inclination) in an heterogeneous population formed by scoliotic and non-scoliotic subjects. The Formetric ${ }^{\circledR}$ system has provided the reference angles.

\section{Study design}

Prospective observational. Fifty three consecutive subjects (nineteen males, thirty-four females, age 16.3 years, thirteen healthy controls/forty scoliosis patients) were measured with the Formetric and the three compared devices in a same session. All measurements by the same observer.

\section{Results}

Mean kyphotic angles measured with the SpineScan (43.8 degrees), the Myrin (34.9 degrees) and Saunders (35.8 degrees) were significantly lower compared with the Formetric (50 degrees). Mean lordotic angles measured with Myrin (32.5 degrees) and Saunders (32.8) were significantly lower compared with Formetric (39.4 degrees). Myrin pelvis inclination (19.4 degrees) was not different compared to the Formetric (20.3 degrees) but both were lower than Saunders (29.8 degrees). Myrin angles were similar to those already published. Both Myrin and Saunders showed a strong correlation with Formetric but not the SpineScan.

\section{Conclusion}

Myrin and Saunders devices can be used in substitution of the Formetric to partially assess the sagittal profile in scoliotic and non-scoliotic subjects with less cost. SpineScan needes further verifications and possibly requires a longer training time. 